\title{
Preoperative chemotherapy with a trastuzumab-containing regimen for a patient with gastric cancer and hepatic metastases
}

\author{
Z.-Y. Li, F. Shan, L.-H. Zhang, Z.-D. Bu, A.-W. Wu, X.-J. Wu, \\ X.-L. Zong, S.-X. Li, X. Ji and J.-F. Ji \\ Department of Surgery, Key Laboratory of Carcinogenesis and Translational \\ Research (Ministry of Education), Peking University School of Oncology, \\ Beijing, China \\ Corresponding author: J.-F. Ji \\ E-mail: jiafuji87@163.com
}

Genet. Mol. Res. 13 (4): 10952-10957 (2014)

Received July 29, 2014

Accepted November 17, 2014

Published December 19, 2014

DOI http://dx.doi.org/10.4238/2014.December.19.17

\begin{abstract}
Gastric cancer is the fourth most common cancer worldwide and the leading cause of tumor-related death in China. Gastric cancer is a heterogeneous disease and therefore requires different treatments based on the subtype. We describe a patient who had gastric cancer with liver metastases. Biopsy and tumor analysis using the HercepTest revealed a human epidermal growth factor receptor 2 (HER2)-positive adenocarcinoma as confirmed by fluorescence in situ hybridization. The patient was treated with a regimen of trastuzumab, oxaliplatin, and S-1 (six cycles). When positron emission tomography findings suggested that the metastases had resolved, the patient underwent surgery. Histopathologically, no cancer cells were observed in the resected hepatic tissue. The patient underwent tumor resection surgery, during which the tumor and gastric lymph nodes with lesions were removed. The patient has remained disease-free for 3 months. Therefore, trastuzumab may be an effective agent in the chemotherapeutic treatment of liver metastases
\end{abstract}


in patients with HER2-positive gastric adenocarcinoma.

Key words: Trastuzumab; Gastric adenocarcinoma; HER2; Liver metastasis; Distal gastrectomy

\section{INTRODUCTION}

Gastric cancer is the fourth most common cancer worldwide and the leading cause of tumor-related death in China (Kamangar et al., 2006). Gastric cancer is a heterogeneous disease that may be divided into subgroups based on histological, anatomical, epidemiological, and molecular features. Distinct molecular drivers and tumor biology, and thus different treatment targets and predictive biomarkers, may be implicated in each subtype. However, there is little evidence in the literature regarding the correlation among these different classifications, particularly the molecular aberrations present in each subtype. Surgery for liver metastases arising from gastric adenocarcinoma is reasonable if a complete resection appears feasible after careful preoperative staging (Sakamoto et al., 2007). Hepatic resection should always be considered as an option for patients with gastric cancer and liver metastases (GCLM). Newer cytotoxic agents, such as S-1, irinotecan, and taxanes, have been linked to promising outcomes in patients with metastases. In the future, adjuvant chemotherapy or molecular targeted therapy may provide significant benefits to patients (Kakeji et al., 2010). In this report, we describe a patient with GCLM whose metastases completely resolved after preoperative chemotherapy with a trastuzumab-containing regimen. We discuss strategies of treatment for GCLM and review the pertinent literature.

\section{CASE}

A 56-year-old woman complained of epigastric pain for 2 months. In October 2010, esophagogastroduodenoscopy revealed a 5-cm ulcer on the anterior wall of the antrum. Biopsy of the ulcer revealed a moderately differentiated adenocarcinoma. The human epidermal growth factor receptor 2 (HER2) status of the tumor was analyzed using the HercepTest (Genzyme, Los Angeles, CA, USA). Membrane-staining intensity and patterns were evaluated using the $0-3+$ scale as illustrated by the HercepTest kit scoring guidelines. Scores of 0 or $1+$ were considered to be negative for HER 2 overexpression; scores of $2+$ were considered to be weakly positive; and scores of $3+$ were considered to be strongly positive. The positive control was selected according to the laboratory manual (Figure 1A). HER2 gene amplification was confirmed by fluorescence in situ hybridization, which demonstrated that HER2/chromosome enumeration probe 17 was amplified in this patient (Figure 1B). The patient had no relevant medical history, and she was taking no medications. She had a normal temperature, heart rate, and blood pressure and normal results on cardiac, pulmonary, abdominal, and digital rectal examinations. Staging workup, involving computed tomography (CT) of the chest, abdomen, and pelvis and positron emission tomography (PET)-CT revealed tumor invasion into the serosa, the presence of perigastric lymphadenopathy, and multiple liver metastases, but no other distant metastases. The clinical stage of the tumor was T4N1M1 (Ajani et al., 2011). 
A

B

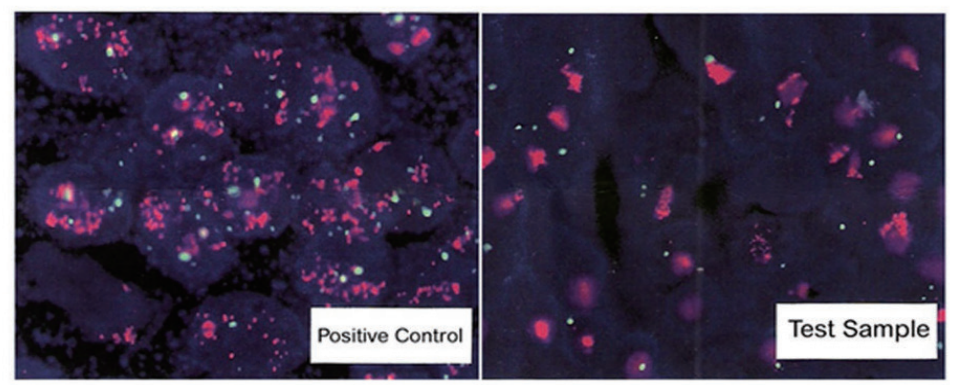

Figure 1. Fluorescence in situ hybridization for human epidermal growth factor receptor 2 (HER2) gene amplification. A. Positive control. B. Numerous fluorescent signals are present in the nucleus of each cell, indicating amplification of the HER2 gene [HER2 signal, red; chromosome enumeration probe 17 signal, green (as an internal control)].

The patient was informed about a trial of trastuzumab in combination with chemotherapy for the treatment of metastatic gastric cancer (ToGA) (Ajani et al., 2011). She agreed to receive a regimen consisting of $6 \mathrm{mg} / \mathrm{kg}$ trastuzumab $i v$ on day $1,100 \mathrm{mg} / \mathrm{m}^{2}$ oxaliplatin $i v$ on day 1 , and $80 \mathrm{mg} / \mathrm{m}^{2} \mathrm{~S}-1$ (TS-1, Taiho Pharmaceutical) orally twice daily on days 1-21, every three weeks. Antiemetics and granulocyte colony-stimulating factor were prescribed as required. She tolerated the regimen well without toxicities of more than grade 2 severity through the completion of cycle 6 . Her tumor response was evaluated and classified on the basis of the criteria defined by the Response Evaluation Criteria in Solid Tumors guidelines (Bang et al., 2010). After two cycles, her response was classified as partial on the basis of CT and PET-CT criteria (Figure 2A). The patient declined our suggestion of surgical resection at that time, hoping that the tumor could be cured without surgery. Thus, she received four additional cycles of treatment. Re-assessment of her tumor status by PET-CT suggested complete remission of the liver metastases, but the gastric lesion remained evident (Figure 2B).
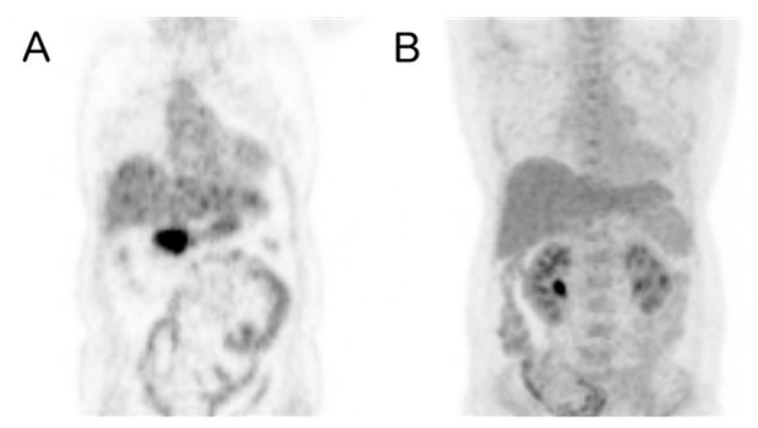

Figure 2. A. Positron emission tomography-computed tomography (PET/CT) image before chemotherapy. B. PET/ CT image after six cycles of treatment.

In May 2011, she underwent distal gastrectomy with D2 lymph node dissection, Billroth-II reconstruction, and partial liver resection. A total of four suspicious lesions (two in $\mathrm{S} 2$, one in $\mathrm{S} 6$, and one in $\mathrm{S} 7$; all $<1 \mathrm{~cm}$ in diameter) were resected from the liver under intraoperative ultrasonic monitoring. Pathological examination of the surgical specimen indicated that an ulcerative type, poorly differentiated, $4 \times 2 \mathrm{~cm}^{2}$ adenocarcinoma was 
located on the lesser curve of the antrum. The tumor had invaded through the serosa, but incisional margins were negative. Carcinoma emboli were observed in the blood vessels. Fatty degeneration, but no cancer cells, was observed in the hepatic nodules. No cancerous cells were identified in 39 lymph nodes. The patient recovered uneventfully after surgery and received two additional cycles of chemotherapy with the same regimen without dose reduction. The patient remains free of disease 3 months after the completion of chemotherapy.

\section{DISCUSSION}

The prognosis of GCLM remains poor, as median survival ranges from 5 to 34 months after the diagnosis of liver metastasis, and survival is shorter if concomitant extrahepatic metastases are present (Therasse et al., 2000; Ambiru et al., 2001; Sakamoto et al., 2007; Cheon et al., 2008). There remains no consensus regarding therapeutic options, and a standard treatment for GCLM has not been established. Hepatic resection for the treatment of metastatic colorectal cancer has been widely employed, but the benefits of gastrectomy and hepatic metastasectomy for synchronous or metachronous metastases from gastric cancer are controversial. A number of other therapeutic options for treating GCLM have been reported, such as hepatic arterial infusion chemotherapy, transcatheter arterial chemoembolization, and thermal ablation; however, the efficacy and safety of these treatments have not been fully evaluated (Koga et al., 2007; Liu and Chen, 2011). Therefore, there is no widely accepted indication or contraindication for the various therapeutic options (Koga et al., 2007).

We previously conducted a pilot study to examine the effect of a weekly regimen of docetaxel and cisplatin plus fluorouracil as a preoperative treatment for gastric cancer with synchronous multiple liver metastases (Li et al., 2010). The regimen had an unprecedented favorable response rate, with all eight treated patients having a positive outcome. However, only one of the eight patients achieved apparent resolution of the hepatic metastases according to imaging studies, thus becoming eligible for hepatic resection of the suspicious lesions. Thus, we consider important that a more effective regimen for GCLM is developed. The ToGA study established a new paradigm for the use of trastuzumab in combination with chemotherapy in patients with HER2-overexpressing advanced gastric cancer (Liu and Chen, 2011). Gastric cancer that has metastasized to the liver has been found to overexpress HER2 at a significantly higher rate than primary gastric cancer (Liu and Chen, 2011). Thus, metastatic liver cancers arising from a primary gastric lesion appear to be suitable targets for gefitinib and trastuzumab therapy (Liu and Chen, 2011). A single case of a patient with locally advanced gastric cancer (no hepatic metastases) who showed complete pathological remission after neoadjuvant chemotherapy with a trastuzumab-containing regimen has been reported (Yokoyama et al., 2006). For these reasons, we used a trastuzumab-containing regimen to treat our patient.

The Medical Research Council Adjuvant Gastric Infusional Chemotherapy trial of perioperative chemotherapy in patients with resectable adenocarcinoma of the stomach, gastroesophageal junction, or distal esophagus was previously conducted (Cunningham et al., 2006; Wang et al., 2010). However, pathological complete responses were not noted in patients who received preoperative epirubicin, cisplatin, and infused fluorouracil in that study.

The administration of trastuzumab can cause subclinical and clinical cardiac failure, and the incidence of this complication is much higher in patients who receive trastuzumab concurrently with anthracycline-containing chemotherapeutic regimens (Cunningham et al., 
2006). For this reason, we selected S-1 and oxaliplatin rather than an anthracycline for our regimen (Cunningham et al., 2008; Li and Chen, 2011; Oh et al., 2012). Earlier studies have demonstrated the therapeutic importance of trastuzumab in combination chemotherapy for the treatment of gastric carcinoma (Park et al., 2010; Amano et al., 2012). Amano et al. (2012) found that combination chemotherapy with trastuzumab, docetaxel, and S-1 in a 74-year-old man with HER2-positive, alpha fetoprotein (AFP)-secreting gastric cancer led to a significant decrease in tumor size and serum AFP levels, with no major adverse events. Nishi et al. (2012) reported a case of a 62-year-old man with HER2-positive scirrhous gastric carcinoma who was treated for 10 cycles with a combination of capecitabine, cisplatin, and trastuzumab. The authors observed complete disappearance of the lesion following this regimen. Ojima et al. (2012) found that the combination of trastuzumab and paclitaxel as second-line therapy in a patient with HER2-positive advanced gastric cancer (type 3) that failed to respond to S-1 and cisplatin combination chemotherapy induced a significant reduction in primary tumor size as well as in the size of the multiple liver metastases. These studies demonstrate the significance of trastuzumab in combination chemotherapy for gastric carcinoma. However, all of the studies used trastuzumab following surgery to remove the primary tumor, and they did not explore its use in patients with liver metastasis.

This is the first reported case of preoperative chemotherapy with a trastuzumab-containing regimen for a patient with HER2-overexpressing GCLM. The promising outcome in our patient should prompt further investigation of the possible efficacy and safety of this comprehensive treatment for GCLM.

\section{Conflicts of interest}

The authors declare no conflict of interest.

\section{ACKNOWLEDGMENTS}

Research supported by the Major Cultivation Project of Beijing Medical Products and Technology (\#Z101107050210027).

\section{REFERENCES}

Ajani JA, Barthel JS, Bentrem DJ, D’Amico TA, et al. (2011). NCCN Clinical Practice Guidelines in Oncology. Gastric Cancer 8: 378-409.

Amano I, Sawai N, Mizuno C, Shaura Y, et al. (2012). A case of HER2-positive and AFP-producing gastric cancer successfully treated by trastuzumab/docetaxel/S-1 combination therapy. Gan To Kagaku Ryoho 39: 2541-2544.

Ambiru S, Miyazaki M, Ito H, Nakagawa K, et al. (2001). Benefits and limits of hepatic resection for gastric metastases. Am. J. Surg. 181: 279-283.

Bang YJ, Van Cutsem E, Feyereislova A, Chung HC, et al. (2010). Trastuzumab in combination with chemotherapy versus chemotherapy alone for treatment of HER2-positive advanced gastric or gastro-oesophageal junction cancer (ToGA): a phase 3, open-label, randomised controlled trial. Lancet 376: 687-697.

Cheon SH, Rha SY, Jeung HC, Im CK, et al. (2008). Survival benefit of combined curative resection of the stomach (D2 resection) and liver in gastric cancer patients with liver metastases. Ann. Oncol. 19: 1146-1153.

Cunningham D, Allum WH, Stenning SP, Thompson JN, et al. (2006). Perioperative chemotherapy versus surgery alone for resectable gastroesophageal cancer. N. Engl. J. Med. 355: 11-20.

Cunningham D, Starling N, Rao S, Iveson T, et al. (2008). Capecitabine and oxaliplatin for advanced esophagogastric cancer. N. Engl. J. Med. 358: 36-46. 
Kakeji Y, Morita M and Maehara Y (2010). Strategies for treating liver metastasis from gastric cancer. Surg. Today 40: $287-$ 294.

Kamangar F, Dores GM and Anderson WF (2006). Patterns of cancer incidence, mortality, and prevalence across five continents: defining priorities to reduce cancer disparities in different geographic regions of the world. J. Clin. Oncol. 24: $2137-2150$.

Koga R, Yamamoto J, Ohyama S, Saiura A, et al. (2007). Liver resection for metastatic gastric cancer: experience with 42 patients including eight long-term survivors. Jpn. J. Clin. Oncol. 37: 836-842.

Li T and Chen L (2011). Efficacy and safety of SOX regimen as neoadjuvant chemotherapy for advanced gastric cancer. Zhonghua Wei Chang Wai Ke. Za Zhi. 14: 104-106.

Li ZY, Tang L, Zhang LH, Bu ZD, et al. (2010). Weekly docetaxel and cisplatin plus fluorouracil as a preoperative treatment for gastric cancer patients with synchronous multiple hepatic metastases: a pilot study. Med. Oncol. 27: 1314-1318.

Liu J and Chen L (2011). Current status and progress in gastric cancer with liver metastasis. Chin Med. J. 124: 445-456.

Nishi T, Hamamoto Y, Uemoto J, Onodera K, et al. (2012). A complete response of scirrhous gastric carcinoma treated with trastuzumab combination therapy. Gan To Kagaku Ryoho 39: 2553-2555.

Oh SY, Kwon HC, Jeong SH, Joo YT, et al. (2012). A phase II study of S-1 and oxaliplatin (SOx) combination chemotherapy as a first-line therapy for patients with advanced gastric cancer. Invest. New Drugs 30: 350-356.

Ojima E, Nishimura G, Nishijima K, Futagami F, et al. (2012). A case of unresectable HER2-positive advanced gastric cancer successfully treated by combination therapy with trastuzumab and paclitaxel. Gan To Kagaku Ryoho 39: 2339-2341.

Park I, Lee JL, Ryu MH, Chang HM, et al. (2010). Phase I/II and pharmacokinetic study of S-1 and oxaliplatin in previously untreated advanced gastric cancer. Cancer Chemother. Pharmacol. 65: 473-480.

Sakamoto Y, Sano T, Shimada K, Esaki M, et al. (2007). Favorable indications for hepatectomy in patients with liver metastasis from gastric cancer. J. Surg. Oncol. 95: 534-539.

Therasse P, Arbuck SG, Eisenhauer EA, Wanders J, et al. (2000). New guidelines to evaluate the response to treatment in solid tumors. European Organization for Research and Treatment of Cancer, National Cancer Institute of the United States, National Cancer Institute of Canada. J. Natl. Cancer Inst. 92: 205-216.

Wang J, Saukel GW, Garberoglio CA, Srikureja W, et al. (2010). Pathological complete response after neoadjuvant chemotherapy with trastuzumab-containing regimen in gastric cancer: a case report. J. Hematol. Oncol. 3: 31.

Yokoyama H, Ikehara Y, Kodera Y, Ikehara S, et al. (2006). Molecular basis for sensitivity and acquired resistance to gefitinib in HER2-overexpressing human gastric cancer cell lines derived from liver metastasis. Br. J. Cancer 95: 1504-1513. 